\title{
The important roles of RET, VEGFR2 and the RAF/ MEK/ERK pathway in cancer treatment with sorafenib
}

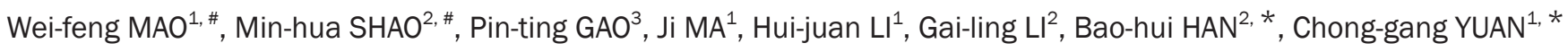 \\ ${ }^{1}$ Biology Department, East China Normal University, Shanghai 200062, China; ${ }^{2}$ Department of Respiratory Disease, Shanghai Chest \\ Hospital, Shanghai Jiao Tong University, Shanghai 200030, China; ${ }^{3}$ Shanghai Medical College (MD Program), Fudan University, \\ Shanghai 200032, China
}

Aim: To elucidate the roles of receptor tyrosine kinases RET and VEGFR2 and the RAF/MEK/ERK signaling cascade in cancer treatment with sorafenib.

Methods: The cell lines A549, HeLa, and HepG2 were tested. The enzyme activity was examined under cell-free conditions using 384well microplate assays. Cell proliferation was evaluated using the Invitrogen Alarmar Blue assay. Gene expression was analyzed using the Invitrogen SYBR Green expression assays with a sequence detection system. Protein expression analysis was performed using Western blotting.

Results: Sorafenib potently suppressed the activities of CRAF, VEGFR2, and RET with IC Fo $_{50}$ values of $20.9,4$, and $0.4 \mathrm{nmol} / \mathrm{L}$, respectively. Sorafenib inhibited cRAF, VEGFR2, and RET via non-ATP-competitive, ATP-competitive and mixed-type modes, respectively. In contrast, sorafenib exerted only moderate cytotoxic effects on the proliferation of the 3 cell lines. The $\mathrm{IC}_{50}$ values for inhibition of A549, HeLa, and HepG2 cells were 8572,4163 , and $8338 \mathrm{nmol} / \mathrm{L}$, respectively. In the 3 cell lines, sorafenib suppressed the cell proliferation mainly by blocking the MEK/ERK downstream pathway at the posttranscriptional level, which in turn regulated related gene expression via a feed-back mechanism.

Conclusion: This study provides novel evidence that protein kinases RET and VEGFR2 play crucial roles in cancer treatment with sorafenib.

Keywords: sorafenib (BAY 43-9006); cancer; protein kinase; VEGFR2; RET; RAF/MEK/ERK pathway

Acta Pharmacologica Sinica (2012) 33: 1311-1318; doi: 10.1038/aps.2012.76; published online 3 Sep 2012

\section{Introduction}

Cancer, known medically as a malignant neoplasm, is responsible for a large number of deaths worldwide. The development of tumor chemotherapy led to the recent discovery of sorafenib (BAY 43-9006). Sorafenib, a multiple kinase inhibitor and novel oral bis-aryl urea compound that exhibits a strong anticancer effect, has been approved by the US Food and Drug Administration for the treatment of advanced renal cell carcinoma and hepatocellular carcinoma ${ }^{[1-5]}$. Many studies suggest that sorafenib could inhibit tumor growth by targeting protein kinases ${ }^{[6-9]}$. In the past few years, accumulating evidence has indicated that protein kinases play critical roles in cancer development, as they are critical components of the cel-

\footnotetext{
* To whom correspondence should be addressed.

E-mail cgyuan@bio.ecnu.edu.cn (Chong-gang YUAN); xkyyhan@gmail.com (Bao-hui HAN)

Received 2012-01-16 Accepted 2012-05-08
}

lular signal transduction cascades; accordingly, kinases have become the most important targets for drug development. The receptor tyrosine kinases VEGFR-2 and RET and the RAF serine/threonine kinases are the important targets of sorafenib in cancer treatment.

VEGFR-2 (Flk-1 in mice and KDR, kinase insert domain-containing receptor, in humans) is an important receptor tyrosine kinase that plays a critical role in tumor angiogenesis, which is an important process for the growth, progression, and metastasis of solid tumors ${ }^{[10]}$. VEGFR-2 can be activated by extracellular signals and then induce downstream signal transduction pathways to regulate cellular activities. In tumors, however, VEGFR-2 is usually found to be aberrantly expressed or constitutively phosphorylated. Thus, VEGFR-2 has become a central focus of molecularly targeted cancer therapy.

Another important protein kinase is the receptor tyrosine kinase RET. This kinase was often found to be abnormally activated in the thyroid, generally through sporadic and 
inherited gene mutations. Hence, it has been hypothesized that the oncogene RET and the RET receptor tyrosine kinase might contribute to the development of cancer ${ }^{[11,12]}$. Indeed, the oncogenic activation of the RET gene is recognized as an early pathogenic event in cancers, and it subsequently induces downstream signaling events involving the MEK/ERK-, PI3K/AKT-, and phospholipase $\mathrm{C}_{Y}$ (PLCY)-dependent pathways $^{[13,14]}$.

The MEK/ERK pathway is also called the RAF/MEK/ERK serine/threonine kinase cascade, as its upstream molecules belong to the RAF kinase family of serine/threonine kinases ${ }^{[15]}$. As a critical messenger, RAF can direct the upstream signal to activate MEK1/2, which, in turn, activates ERK1/2 ${ }^{[16,17]}$. Activated ERK1 or ERK2 then either phosphorylates its target proteins in the cytoplasm or translocates to the nucleus, where the main targets are transcription factors that regulate proliferation-, differentiation- or survival-related genes. Therefore, the RAF/MEK/ERK serine/threonine kinase cascade is a key pathway that is involved in the development of cancers and is considered a potential important target for sorafenib treatment ${ }^{[18]}$.

However, the mechanism by which sorafenib inhibits tumor cells and how kinases respond to sorafenib treatment are largely unclear. Therefore, this study aims to elucidate the events at the biomolecular level in three cell lines (A549, HeLa, and HepG2) after sorafenib treatment. Because different cancers might be regulated by different mechanisms, we selected three cell lines that have different metastatic potential, and enzyme kinetic, cytology, RT-PCR and Western blotting assays were conducted. Specifically, one serine/threonine kinase, cRAF, and two tyrosine kinases, VEGFR2 and RET, and their potential downstream MEK/ERK pathway targets were examined. An understanding of the molecular events in cancer cells treated with sorafenib not only is necessary to obtain a better insight into genotype-phenotype correlations but also is crucial for the discovery of new therapeutic approaches.

\section{Materials and methods Compounds}

Sorafenib was produced by the Chemistry Department, Chinese Pharmaceutical University (Nanjing, China); staurosporine, PI-103, and mitomycin C were purchased from Sigma Aldrich, Germany.

\section{Kinases}

cRAF (Y340D and Y341D) and VEGFR2 (KDR) were purchased from Invitrogen. RET was expressed in Sf9 cells using an Invitrogen baculovirus system and then purified by His/ GST affinity and Supdex35/75/200 chromatography. The recombinant proteins were then analyzed by SDS-PAGE, and the molecular weights were determined using LS-MS/MS, model API3000.

\section{Activity assays}

Using 384-well microplate assays, an enzyme titration, the
ATP $K_{\mathrm{m}}$, a time course, and a positive-control $\mathrm{IC}_{50}$ were employed to validate the kinases. The CRAFs were analyzed using the Invitrogen LanthaScreen Tb-activity assay with 0.01 $\mathrm{nmol} / \mathrm{L}$ kinase, $200 \mathrm{nmol} / \mathrm{L}$ Fluorescein-MAP2K1, $50 \mathrm{mmol} / \mathrm{L}$ HEPES (pH 7.5), $10 \mathrm{mmol} / \mathrm{L} \mathrm{MgCl}_{2}$ and $0.01 \%$ Brij35 for $1 \mathrm{~h}$ at $23^{\circ} \mathrm{C}$; the reaction was terminated with $30 \mathrm{mmol} / \mathrm{L}$ EDTA and $2 \mathrm{nmol} / \mathrm{L}$ phospho-MAP2K1 antibody. Cisbio HTRF technology was employed to analyze the activity of optimized concentrations of RET and VEGFR2, with $50 \mathrm{mmol} / \mathrm{L}$ HEPES ( $\mathrm{pH}$ 7.5), $10 \mathrm{mmol} / \mathrm{L} \mathrm{MgCl}_{2}$, ATP $K_{\mathrm{m}}, 0.01 \%$ Brij35, and $500 \mathrm{nmol} /$ L STK1 peptide for $1 \mathrm{~h}$ at $23^{\circ} \mathrm{C}$. For the $\mathrm{IC}_{50}$ test, the sorafenib concentration was $10 \mu \mathrm{mol} / \mathrm{L}$ for 11 doses in duplicate, with 3 -fold dilutions of ATP $K_{\mathrm{m}}$. For the MOA study, 6 ATP concentrations $\left(1 / 3-, 1-, 3-, 9-, 27-\right.$, and 81-fold ATP $\left.K_{\mathrm{m}}\right)$ were used at the same serial dilution as in the $\mathrm{IC}_{50}$ test. The readouts were analyzed using the PerkinElmer Envision2104 reader in Htrf/FRET module. The Inh\% was analyzed using Excel. The $\mathrm{IC}_{50}$ Shift and the Lineweaver-Burk and double-reciprocal plots were drawn using Graphpad Prism 5.

\section{Cell culture}

The A549, HeLa and HepG2 cells were obtained from ATCC. A549 was cultured in F12, 10\% FBS, and 2 mmol/L glutamine at $37^{\circ} \mathrm{C}$ under $5 \% \mathrm{CO}_{2}$; the HeLa and HepG2 cells were cultured in DMEM-HG, $10 \% \mathrm{FBS}$, and $2 \mathrm{mmol} / \mathrm{L}$ glutamine.

\section{Cell proliferation}

The cells were detached using accutase (Millipore) and counted using trypan blue staining and a ViaCell (Beckman Coulter). The A549 $\left(5 \times 10^{3}\right.$ cells $)$, HeLa $\left(3 \times 10^{3}\right.$ cells $)$, and HepG2 $\left(1 \times 10^{4}\right.$ cells $)$ cells were then plated in triplicate in 96-well microplate in the presence of sorafenib, mitomycin C, or vehicle (DMSO, $0.2 \%$ ) for $0,24,48$, and $72 \mathrm{~h}$. The cell proliferation assay was evaluated using Invitrogen Alarmar Blue by adding $10 \mu \mathrm{L}$ of the reagents after $96 \mathrm{~h}$.

\section{RT-PCR}

The A549 $\left(5 \times 10^{3}\right)$, HeLa $\left(3 \times 10^{3}\right)$ and HepG2 $\left(1 \times 10^{4}\right)$ cells were plated in 30-mm dishes for 24,48 , and $72 \mathrm{~h}$, and each cell line $\left(5 \times 10^{5}\right)$ was also plated for 2,4 , and $8 \mathrm{~h}$. Sorafenib at $5 \mu \mathrm{mol} / \mathrm{L}$ or $1 \mu \mathrm{mol} / \mathrm{L}$ or vehicle (DMSO, $0.2 \%$ ) were applied for 2,4 , and $8 \mathrm{~h}$ as described above.

The RNA extraction, cDNA synthesis, and quantitative realtime PCR amplification were performed using the RNeasy kit (Qiagen) according to the manufacturer's instructions. For the cDNA synthesis, $200 \mathrm{ng}$ of mRNA was used with the Omniscript RT kit (Qiagen), following the manufacturer's instructions, with oligo (dT)20 primers (Qiagen). A 1- $\mu$ g sample of cDNA was used for each qPCR, which was performed in triplicate.

Primers for ERK (forward 5'-3', GCGCTACACCAACCTCTCGT; reverse 5' $-3^{\prime}$, CACGGTGCAGAACGTTAGCTG), VEGFR2 (forward 5'-3', CACCACTCAAACGCTGACATGTA; reverse 5'-3', GCTCGTTGGCGCACTCTT), RAF1 (forward 5'-3', GAACGACAGGACGTTGGG; reverse 5' $-3^{\prime}$, GCTGATCGTCTTCCAAGCTC), and RET (forward 5' $-3^{\prime}$, 
CTAGCCGCAGTCCCTCC; reverse 5'-3', AAGCATCCCTCGAGAAGTAGA), were designed for the RT-PCR. GAPDH, $\mathrm{ACTB}$, and $\mathrm{B} 2 \mathrm{M}$ were used as the endogenous controls in all of the experimental analyses. Invitrogen Sybgreen expression assays and an ABI Prism 7900HT sequence detection system (Applied Biosystems, Foster City, CA, USA) were employed for the gene expression analyses with the $\Delta \Delta \mathrm{Ct}$ method, which determines the fold change in gene expression relative to a comparator sample (control). The PCR primers were purchased from Shanghai Generay Biotech Company.

Effects of sorafenib on three cell lines at the transcriptional level To demonstrate the effect of sorafenib on cells at the transcription level, the mRNA concentrations of four genes, RET, VEGFR2, cRAF and ERK, were detected using RT-PCR assays. Each cell line was treated with sorafenib at two concentrations, 1 and $5 \mu \mathrm{mol} / \mathrm{L}$, and the cells were collected after 2, 4, and $8 \mathrm{~h}$; cells without sorafenib treatment were used as the controls $(0 \mathrm{~h})$. The total mRNA was extracted and quantified for use in RT-PCR assays. The ratio of the target mRNA content in the treatment group to the controls was calculated.

\section{Western blotting}

After their respective treatments, the cells were lysed in lysis solution (Cell Signaling Technology, Danvers, MA, USA) supplemented with sodium fluoride $(10 \mathrm{~mol} / \mathrm{L}$; Fisher) and phenylmethylsulfonyl fluoride (100 g/ mL; Sigma-Aldrich). The lysates were centrifuged at $14000 \times g$ for $30 \mathrm{~min}$, and the supernatants were aspirated to determine the protein concentration using BCA reagents (Pierce, ThermoFisher, Rockford, IL, USA). Each $80 \mu \mathrm{g}$ sample was fractionated in either $8 \%$ or $12 \%$ SDS-polyacrylamide gels, and the separated proteins were transferred to nitrocellulose. The blots were probed for the proteins of interest using primary antibodies followed by a secondary antibody-horseradish peroxidase conjugate. The Super Signal chemiluminescence substrate (Pierce) and the GE Image Quant analyzer were used for the detection.

The anti-ERK (total) and anti-RET (pY1062) antibodies were obtained from Santa Cruz Biotechnology (Santa Cruz, CA, USA). Anti-Erk (pT202/pY204), anti-VEGFR2 (pY1214), antiRET (pY1016), anti-MEK1 (pT292), and anti-MEK1 (p298) were obtained from Invitrogen. Anti-VEGFR2 (total), anti-VEGFR2 (pY1175), anti-RET (total), anti-RET (pY905), and anti-MEK (total) were purchased from Cell Signaling.

\section{Effects of sorafenib on the three cell lines at the post-trans- criptional level}

To investigate the effect of sorafenib on the post-transcriptional events in cells, the expression and phosphorylation level of the following four proteins were detected by Western blot assays: VEGFR2, RET, MEK, and ERK. Each cell line was treated with two concentrations of sorafenib, 1 and $5 \mu \mathrm{mol} / \mathrm{L}$, and the cells were then collected after 2,4 , and $8 \mathrm{~h}$. Cells without sorafenib treatment were used as the controls $(0 \mathrm{~h})$. The total proteins were extracted and quantified in Western blot assays.

\section{Statistical analysis}

All statistical analysis was taken under student $t$-test with SPSS 18.0. $P<0.05$ was considered statistically significant for two-side $P$-value test.

\section{Results}

Sorafenib inhibits the activity of kinases by different inhibition mechanisms under cell-free conditions

The effect of sorafenib on enzyme activity was measured by assays one hour after the drug was applied. Sorafenib inhibited the activity of $\mathrm{CRAF}$, with an $\mathrm{IC}_{50}$ of $20.9 \mathrm{nmol} / \mathrm{L}$ and 0.4 $\mathrm{nmol} / \mathrm{L}$ and $4 \mathrm{nmol} / \mathrm{L}$ for RET and VEGFR2, respectively. The data for $\mathrm{CRAF}$ agreed with the previous report of an $\mathrm{IC}_{50}$ of $19 \mathrm{nmol} / \mathrm{L}$. In contrast, the results for VEGFR2 and RET were quite different from the reported data of $90 \mathrm{nmol} / \mathrm{L}$ and $4 \mathrm{nmol} / \mathrm{L}$, respectively (Figure 1). The double-reciprocal plot shows that the linear regression alignments converge on the $Y$ axis (Figure 2A, 2C), indicating that sorafenib is an ATP-competitive compound for RET and KDR, a result that is consistent with Wilhelm's ${ }^{[8]}$. However, this effect was not observed for cRAF (Figure 2E), and, when ATP was added, the $\mathrm{IC}_{50}$ for cRAF was consistent with the different concentrations (1/3-, 1-, 3-, 9-, 27-, and 81-fold ATP $K_{m}$ ) (Figure 2F), which indicated that sorafenib inhibited $\mathrm{CRAF}$ activity through a non-ATPcompetitive mode. For KDR/VEGFR2, the $\mathrm{IC}_{50}$ rose consistently with the increase in the ATP concentration (Figure 2D), suggesting that sorafenib inhibited the KDR/VEGFR2 activity through an ATP-competitive mechanism. For RET, the $\mathrm{IC}_{50}$ improved with the increase in the ATP concentration in a nonhomogeneous manner (Figure 2B), which demonstrated that sorafenib inhibited the RET activity through a mixed-type inhibition. In summary, sorafenib targets the ATP-binding site of the kinases and inhibits their activities by different inhibition mechanisms under cell-free conditions.

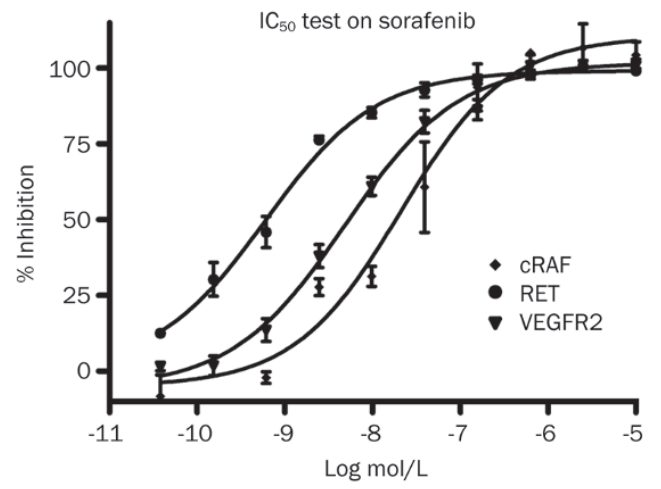

\begin{tabular}{lcc}
\hline Target name & Reported IC $\mathrm{C}_{50}(\mathrm{nmol} / \mathrm{L})$ & Test IC $_{50}(\mathrm{nmol} / \mathrm{L})$ \\
\hline CRAF & $19 \pm 5.7$ & 20.9 \\
KDR/VEGFR2 & $90 \pm 27$ & 4 \\
RET & $4 \pm 0.8$ & 0.4 \\
\hline
\end{tabular}

Figure 1. Sorafenib inhibits kinase activity of cRAF, RET, and VEGFR2 with different $\mathrm{IC}_{50}$ under cell-free condition. 

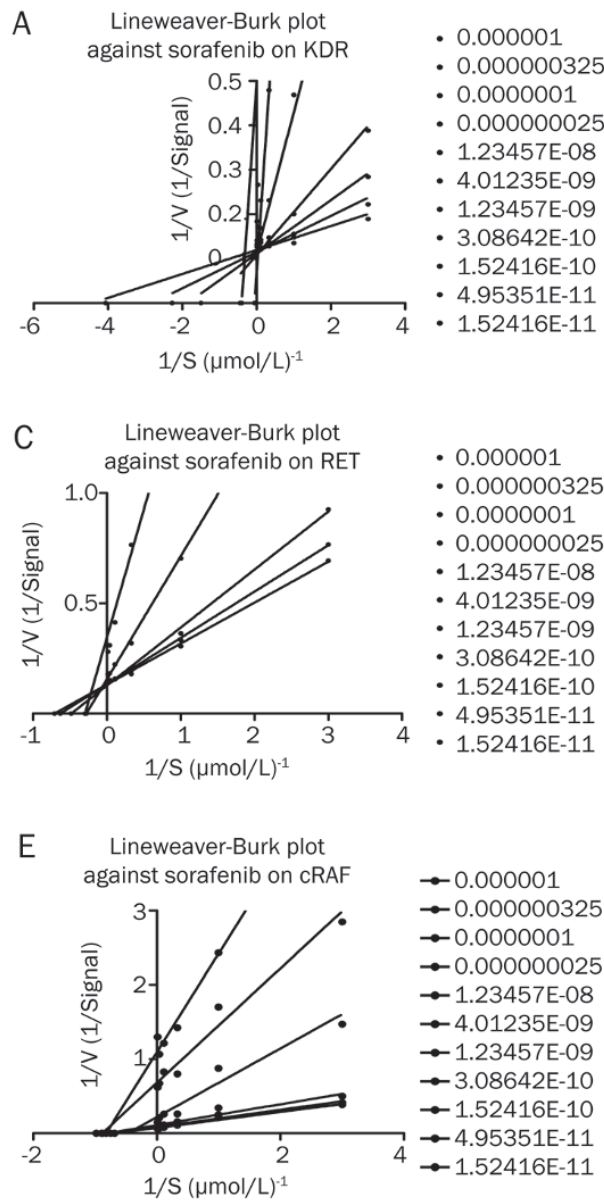
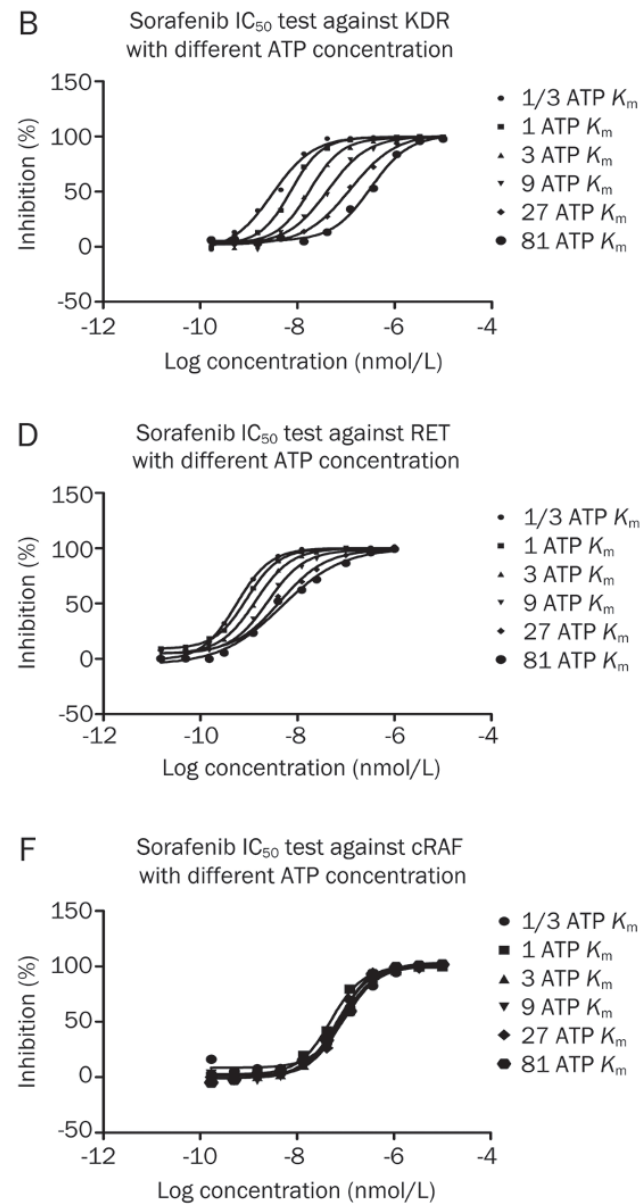

Figure 2. Double-reciprocal plot of kinetic data from assays of KDR, RET, and CRAF protein kinase activity at different concentrations of sorafenib. ATP concentrations in the reaction mixture varied from $1 / 3$ ATP $K_{m}$ to 81 fold ATP $K_{m}$. Sorafenib concentrations are from $100 \mu \mathrm{mol} / \mathrm{L}$ to $1.6 \mathrm{nmol} / \mathrm{L}$ with 3 -fold dilution and the concentration of peptides were kept constant at $2 \mu \mathrm{mol} / \mathrm{L}$. $V$ is picomoles of phosphate/60 min. Double-reciprocal plot shows the linear regression alignments are converge on $\mathrm{Y}$ axis, which means sorafenib is an ATP competitive compound on KDR, RET, which is consistent with reported. The $\mathrm{IC}_{50}$ of $\mathrm{RET}, \mathrm{KDR}$ are $0.4 \mathrm{nmol} / \mathrm{L}, 4 \mathrm{nmol} / \mathrm{L}$ in sequence.

Sorafenib inhibits proliferation in A549, HeLa and HepG2 cells The effect of sorafenib on cell proliferation was examined at $72 \mathrm{~h}$ in the A549, HeLa, and HepG2 cell lines. Sorafenib displayed a moderate dose-dependent inhibition of cell proliferation. The $\mathrm{IC}_{50}$ values for the $\mathrm{A} 549, \mathrm{HeLa}$, and HepG2 cells were $8572 \mathrm{nmol} / \mathrm{L}, 4163 \mathrm{nmol} / \mathrm{L}$, and $8338 \mathrm{nmol} / \mathrm{L}$, respectively, and the maximum inhibition was $88 \%, 78 \%$, and $94 \%$ for each of the three cell lines, respectively. Figure 3 shows the inhibition of cell proliferation by $20 \mu \mathrm{mol} / \mathrm{L}$ sorafenib in the three cell lines compared with the cells treated with DMSO (controls).

Sorafenib activates RET expression and inhibits VEGFR2 expression independent of the RAF/MEK/ERK pathway at the transcriptional level in A549 cells

In the A549 cells, the expression of RET increased significantly at $2 \mathrm{~h}$ after treatment with $1 \mu \mathrm{mol} / \mathrm{L}$ sorafenib and then decreased, whereas the VEGFR2 expression notably decreased in every treatment group (Figure 4A). In contrast, the cRAF and ERK expression levels were not significantly affected, even though the cRAF expression showed a slight decrease at $5 \mu \mathrm{mol} / \mathrm{L}$ sorafenib. These results suggested that sorafenib could activate RET expression and inhibit VEGFR2 expression independent of the RAF/MEK/ERK pathway at the transcriptional level in A549 cells.

Sorafenib inhibits VEGFR2 expression but has no effect on the RAF/MEK/ERK pathway at the transcriptional level in HeLa cells

The same assay as described above for the A549 cells was performed using HeLa cells. The results showed that sorafenib up-regulated the expression of RET; conversely, sorafenib significantly down-regulated the expression of VEGFR2 at the transcription level (Figure 4B). A similar result was found for the HeLa cells in that sorafenib showed no effect on cRAF and ERK expression. These results suggested that sorafenib could inhibit VEGFR2 expression but had no effect on the RAF/ MEK/ERK pathway at the transcriptional level in HeLa cells. 

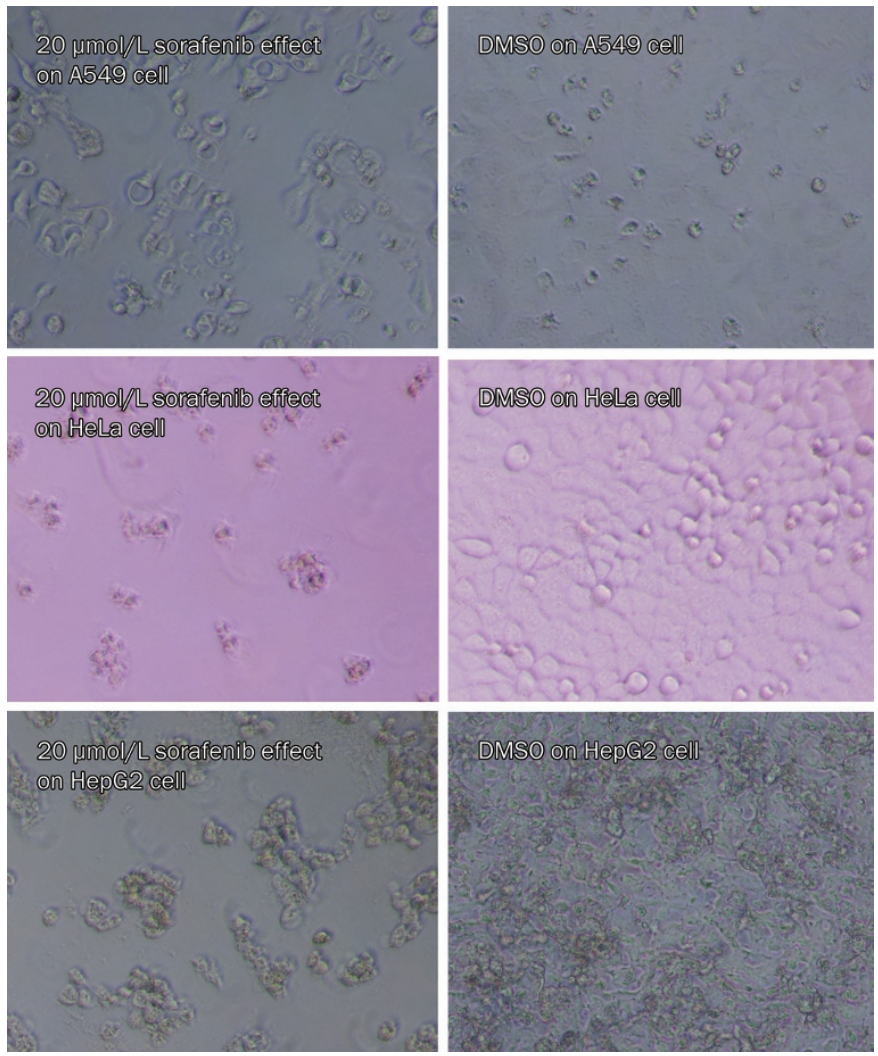

Figure 3. Sorafenib moderately inhibited proliferation of A549, HeLa and HepG2 cells. Sorafenib was added to A549, HeLa, and HepG2 cells, and cultured for $72 \mathrm{~h}$ in culture medium, meanwhile, DMSO was added to controls. Sorafenib displayed moderate cytotoxicity to cell proliferation dose-dependently.

Sorafenib inhibits RET expression dose-dependently and activates VEGFR2 expression dose- and time-dependently but has no effect on the RAF/MEK/ERK pathway at the transcriptional level in HepG2 cells

The results for the HepG2 cell line showed that RET expression was dose-dependently down-regulated by sorafenib at the transcriptional level. The expression of VEGFR2 was initially up-regulated and then down-regulated with $1 \mu \mathrm{mol} / \mathrm{L}$ sorafenib, but it was persistently activated in a time-dependent manner at $5 \mu \mathrm{mol} / \mathrm{L}$ (Figure $4 \mathrm{C}$ ). As in the other two cell lines, sorafenib showed no effect on CRAF and ERK expression. These results suggested that sorafenib dose-dependently inhibited RET expression and dose- and time-dependently activated VEGFR2 expression but had no effect on the RAF/ MEK/ERK pathway at the transcriptional level in HepG2 cells.

Sorafenib directly inhibits RET and VEGFR2 phosphorylation and blocks the downstream signaling of the MEK/ERK pathway at the post-transcriptional level in A549 cells

The results for the A549 cells indicated a significant dosedependent decrease of RET and VEGFR2 phosphorylation (Figure 5A) but showed a notable dose-dependent increase
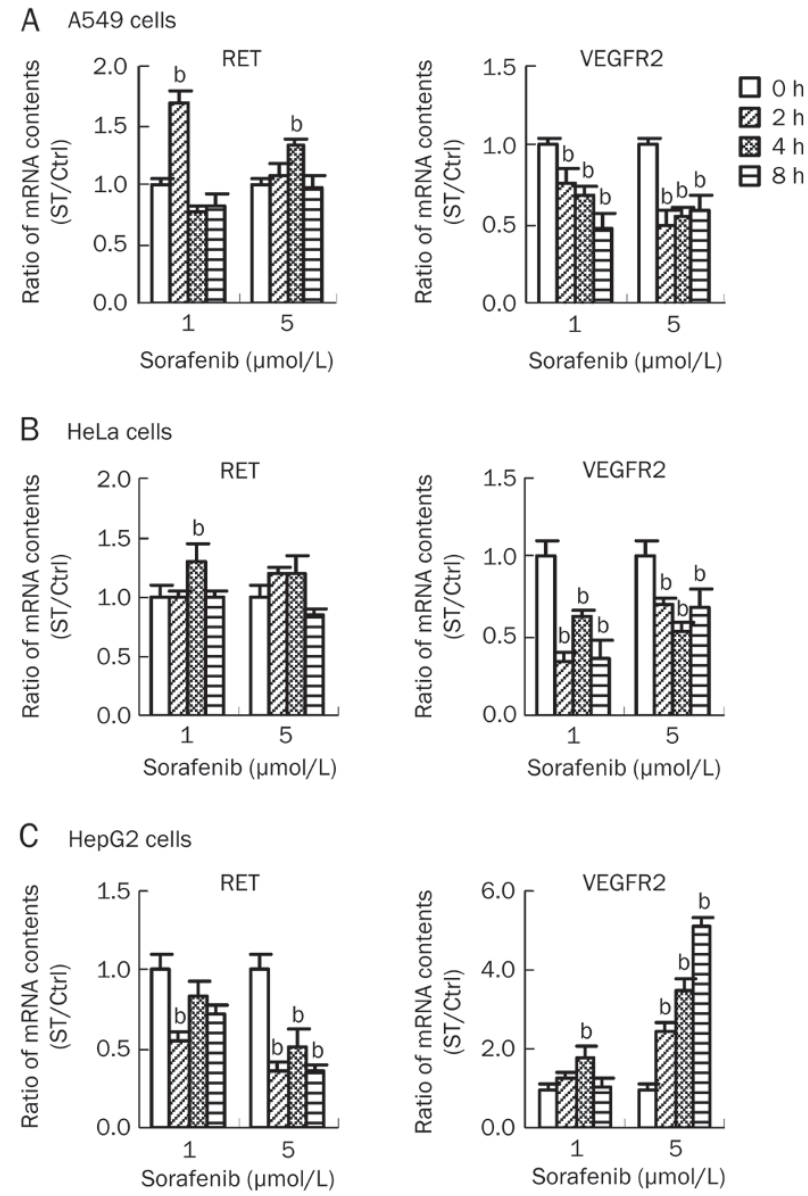

Figure 4. Sorafenib affected RET and VEGFR2 gene expression in A549, HeLa, and HepG2 cells. Three cell lines were treated by sorafenib with two concentration gradients, 1 and $5 \mu \mathrm{mol} / \mathrm{L}$, and then collected after 2 , 4 , and $8 \mathrm{~h}$. Cells without sorafenib treatment were as the controls $(0 \mathrm{~h})$. Total mRNA was extracted and quantified to be used in RT-PCR assays. Ratio for mRNA content in treatment group compared with controls was calculated. (A) Sorafenib up-regulated RET gene expression and down-regulated VEGFR2 gene expression in A549 cells. (B) Sorafenib up-regulated RET gene expression and down-regulated VEGFR2 gene expression in HeLa cells which was similar to that in A549 cells. (C) Sorafenib down-regulated RET gene expression and up-regulated VEGFR2 gene expression in HepG2 cells which opposite to that in the above two cell lines.

of RET and VEGFR2 expression. These findings suggest that sorafenib directly inhibited RET and VEGFR2 phosphorylation, altering the ratio of phosphorylated to unmodified proteins, which then led to an increase in RET and VEGFR2 expression. We found that sorafenib dose-dependently upregulated the expression and activated the phosphorylation of MEK and ERK (Figure 5A), which indicated that sorafenib might inhibit the downstream targets of the MEK/ERK pathway. The increase in the expression and phosphorylation of MEK and ERK can be explained by a feedback regulation mechanism. Therefore, we concluded that sorafenib acts by directly inhibiting the phosphorylation of RET and VEGFR2 and blocking the MEK/ERK downstream pathway at the post- 


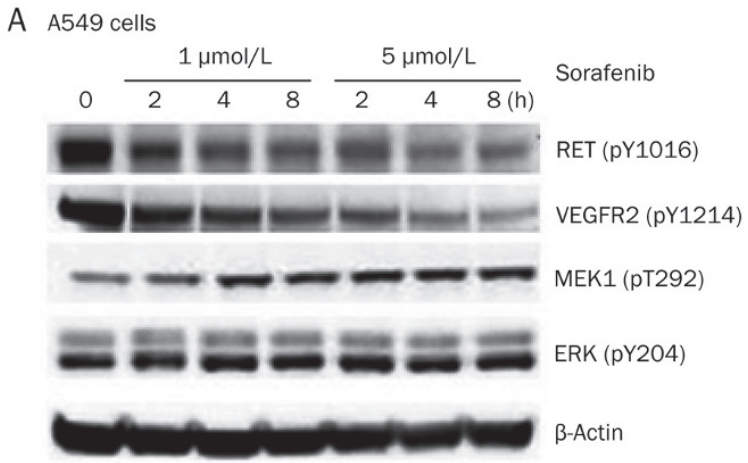

B HeLa cells

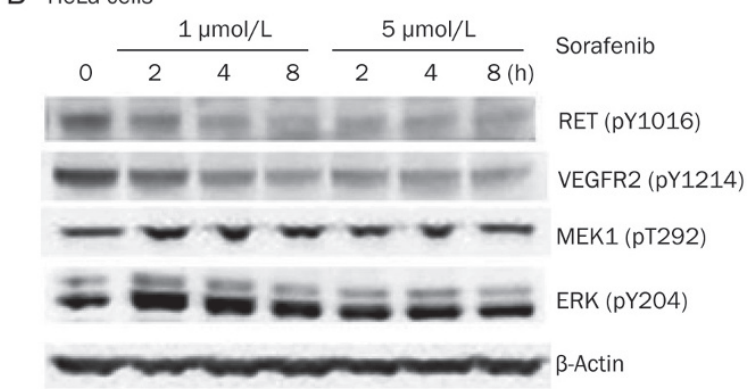

C HepG2 cells

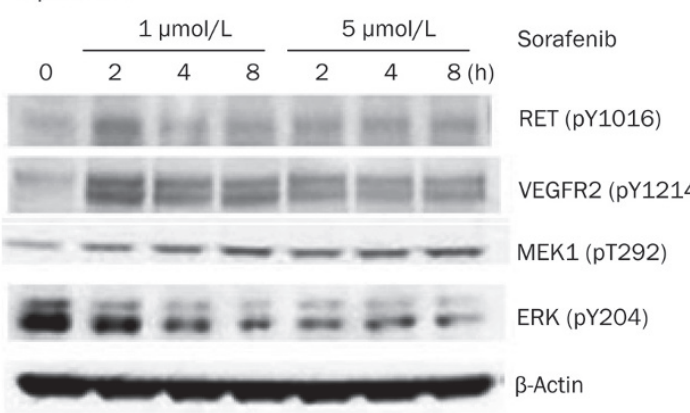

Figure 5. Sorafenib affected the phosphorylation of receptor tyrosine kinase RET and VEGFR2, as well as MEK/ERK kinases signaling cascades in three cell lines. Three cell lines were treated by sorafenib with two concentration gradients, 1 and $5 \mu \mathrm{mol} / \mathrm{L} / \mathrm{L}$, and then collected after 2 , 4 , and $8 \mathrm{~h}$, cells without sorafenib treatment were as the controls $(0 \mathrm{~h})$. Total proteins were extracted and quantified to be used in Western blot assays. (A) Sorafenib inhibited RET and VEGFR2 phosphorylation dosedependently while activated MEK and ERK phosphorylation in A549 cells. (B) Sorafenib also inhibited RET and VEGFR2 phosphorylation, and slightly activated MEK and ERK phosphorylation in HeLa cells. (C) Sorafenib activated the phosphorylation of RET, VEGFR2, and MEK, but inhibited ERK phosphorylation in HepG2 cells.

transcriptional level in A549 cells.

Sorafenib directly inhibits RET and VEGFR2 phosphorylation and blocks the downstream MEK/ERK pathway at the posttranscriptional level in HeLa cells

In HeLa cells, RET and VEGFR2 phosphorylation also decreased notably, as in the A549 cells (Figure 5B), which suggested that sorafenib also directly inhibited the phospho- rylation of RET and VEGFR2 in HeLa cells. The MEK and ERK phosphorylation level first increased and then decreased (Figure 5B), indicating that sorafenib targeted the downstream signaling molecules of the MEK/ERK pathway. The observed mechanism was similar to that in the A549 cells. Consequently, sorafenib directly inhibited RET and VEGFR2 phosphorylation and blocked the MEK/ERK downstream pathway at the post-transcriptional level in HeLa cells.

Sorafenib targets the RET and VEGFR downstream molecules and blocks the MEK/ERK pathway by inhibiting ERK phosphorylation at the post-transcriptional level in HepG2 cells

In the HepG2 cells treated with sorafenib, the expression of RET was little altered, but its phosphorylation level rose at $2 \mathrm{~h}$ after treatment and then decreased to a low level (Figure 5C). This result suggested that sorafenib might target the downstream molecule of RET, disrupting the balance of the related pathways. VEGFR2 phosphorylation significantly increased after sorafenib treatment, which also suggested that sorafenib might target the downstream molecule of VEGFR2 (Figure 5C). Regarding the MEK/ERK pathway, ERK phosphorylation was significantly inhibited by sorafenib, which led to a notable increase in the upstream MEK phosphorylation (Figure 5C), demonstrating that sorafenib blocked the MEK/ERK pathway by directly inhibiting ERK phosphorylation. Therefore, in the HepG2 cell line, sorafenib targeted the downstream molecules of RET and VEGFR2, while blocking the MEK/ERK pathway by inhibiting ERK phosphorylation at the post-transcriptional level.

\section{Discussion}

Receptor tyrosine kinases play major roles in the development of cancer and represent effective targets for specific therapeutic approaches. Indeed, receptor kinase-based therapies have attained wide-spread clinical use, for example, in breast cancer (the inhibition of HER2 by Herceptin) ${ }^{[19]}$, in gastrointestinal stromal tumors (the inhibition of c-Kit by Gleevec) ${ }^{[20,21]}$, and in non-small cell lung cancer (the inhibition of EGF receptor by Gefitinib) ${ }^{[22]}$. Recently, the orally administered multikinase inhibitor sorafenib was found to exert a prominent activity against tumors. Thus, to verify the anticancer mechanism of sorafenib, we performed a series of trials in vitro.

In the present study, we found that sorafenib has a higher $\mathrm{IC}_{50}$ than that described by Wilhelm et al ${ }^{[8]}$, with $\mathrm{IC}_{50}$ values of $20 \mathrm{nmol} / \mathrm{L}$ against cRAF in vitro under cell-free conditions. However, the drug shows a more potent inhibition of VEGFR-2, at $\mathrm{IC}_{50}=4 \mathrm{nmol} / \mathrm{L}$, than that reported by Wilhelm et al, at $\mathrm{IC}_{50}=90 \mathrm{nmol} / \mathrm{L}^{[8]}$. In addition, RET was significantly inhibited by sorafenib, with an $\mathrm{IC}_{50}=0.4 \mathrm{nmol} / \mathrm{L}$, much lower than the value reported by Wilhelm et al, at $\mathrm{IC}_{50}=4 \mathrm{nmol} / \mathrm{L}$. Iván Plaza-Menacho also found that sorafenib inhibited the activity of the purified recombinant kinase domain of widetype RET and the mutant $\mathrm{RET}^{\mathrm{V} 804 \mathrm{M}}$, with $\mathrm{IC}_{50}$ values of 5.9 and $7.9 \mathrm{nmol} / \mathrm{L}$, respectively, which also suggested that sorafenib is an inhibitor of $\mathrm{RET}^{[23]}$.

Several therapeutic avenues are available to block the activ- 
ity of protein kinases. Drugs that bind reversibly to the ATPbinding site within the kinase domain or to a small pocket that is immediately adjacent to the ATP-binding site are used to block the enzymatic activity of the kinase. Because of similarities within the three-dimensional structure of the kinase domain, ATP-competitive inhibitors can have cross-reactivity with other structurally related kinases. The data obtained in the present study indicate that sorafenib is an ATP-competitive inhibitor of VEGFR2 and RET but not cRAF: sorafenib inhibited the activities of CRAF, VEGFR2 and RET through non-ATP-competitive, ATP-competitive and mixed-type inhibition modes, respectively. In summary, sorafenib could target the ATP-binding site of kinases and inhibit the activity of kinases by different inhibition mechanisms under cell-free conditions. This is a novel discovery, and further studies will be conducted to verify the conclusions.

As different cancers might be regulated by different mechanisms, we selected three cell lines having different metastatic potentials (A549, HeLa, and HepG2). At the transcriptional level, we found that sorafenib could briefly activate RET expression and inhibit VEGFR2 expression independent of the RAF/MEK/ERK pathway in A549 cells; at the post-transcriptional level, sorafenib directly inhibited the phosphorylation of RET and VEGFR2 and blocked the downstream factors of the MEK/ERK pathway. Therefore, we hypothesized that sorafenib inhibited the proliferation of A549 cells through anti-proliferation and anti-angiogenesis processes. Moreover, whereas the anti-proliferation process is a post-transcriptional event, anti-angiogenesis refers to many molecular processes from transcription to post-translational modification. The two kinases RET and VEGFR2 could be the targets of sorafenib treatment, but they may not be the upstream messengers of the MEK/ERK kinases pathway. The downstream signaling molecules of the MEK/ERK kinase pathway are also potential candidates for the sorafenib treatment of lung cancer.

According to the results for HeLa cells, we found that sorafenib could inhibit VEGFR2 expression but had no effect on the RAF/MEK/ERK pathway at the transcriptional level. At the post-transcriptional level, sorafenib directly inhibited RET and VEGFR2 phosphorylation and blocked the downstream factors of the MEK/ERK pathway. Therefore, the mechanism of the sorafenib-mediated inhibition of proliferation in HeLa cells is similar to that of A549 cells. RET, VEGFR2 and the downstream signaling molecules of the MEK/ERK kinase pathway are also important potential targets for sorafenib in the treatment of cervical cancer.

In contrast to the results in the above cell lines, sorafenib inhibited RET expression and activated VEGFR2 expression in the HepG2 cells, with little effect on the RAF/MEK/ERK pathway at the transcriptional level. Moreover, sorafenib targeted the downstream molecules of RET and VEGFR2 and blocked the MEK/ERK pathway by inhibiting ERK phosphorylation at the post-transcriptional level. It is clear that anti-proliferation and anti-angiogenesis pathways are also involved in the response to sorafenib treatment in hepatic cancer. The mechanism, however, differs from lung and liver cancer, which might be attributable to the similar origin of lung and liver tissues.

Our results indicated that sorafenib is an inhibitor of the phosphorylation of protein kinase RET and VEGFR2 under cell-free conditions as well as in A549 and HeLa cells. In HepG2 cells, sorafenib markedly inhibited kinase ERK phosphorylation, though the drug did not exhibit a significant inhibition on the phosphorylation of RET and VEGFR2. In addition, based on the present results, the MEK/ERK kinase signaling cascade is clearly an important effective target pathway for cancer treatment. Therefore, it is confirmed that kinases indeed play crucial roles in the effective mechanism of sorafenib and are important effective targets for specific therapeutic approaches. Moreover, sorafenib inhibited cell proliferation mainly by blocking post-translational modifications, which then regulated the related gene expression via a feed-back mechanism. Interestingly, we found that the two kinases, RET and VEGFR2, displayed similar bimolecular alterations, even though they acted in the reaction to sorafenib treatment through different mechanisms in the three cell lines, a finding that could be explained by the results of mechanism of action (MOA) that sorafenib is an ATP-competitive inhibitor of VEGFR2 and RET. In addition, we hypothesized that RET and VEGFR2 were involved in a similar signaling pathway due to their similar biomolecular alteration in the different cell lines treated with sorafenib.

In summary, we found that sorafenib was effective in inhibiting the proliferation of cancer cells by inhibiting kinases through different competitive mechanisms. The receptor tyrosine kinases RET and VEGFR2 and the MEK/ERK kinase signaling cascade are important targets for cancer treatment with sorafenib, even though these factors participate in different pharmacological mechanisms in different cell lines. Furthermore, according to their similar biomolecular alteration in the different cell lines treated with sorafenib, kinases RET and VEGFR2 may be involved in a similar signaling pathway. Therefore, the present study was consistent with previous results and provided more evidence for kinases as the targets in cancer therapy and partially revealed the pharmacological mechanism, including molecules downstream of the MEK/ ERK kinase pathway and the pathways initiated by RET or VEGFR2, of sorafenib in cancer treatment. However, there are still many aspects of the mechanisms that remain to be elucidated. In future studies, we will perform kinase spectrum screening to identify additional protein kinases involved in the mechanisms of cancer treatment, such as the downstream molecules of the MEK/ERK kinase pathway and the pathways in which RET and VEGFR2 are involved.

\section{Acknowledgements}

This work was supported by grants from the Science and Technology Commission of Shanghai Municipality in China (№ O5JC14068 and 075407059).

\section{Abbreviations}

RET, the rearranged during transfection receptor; Raf, v-raf 
1 murine leukemia viral oncogene homolog 1; VEGFR2, vascular endothelial growth factor receptor 2; KDR, kinase insert domain receptor; MEK, mitogen-activated protein kinase; ERK, extracellular signal-regulated kinase; PI3K, phosphatidylinositol 3-kinase; AKT, protein kinase B; PLC $\gamma$, phospholipase $\mathrm{C}_{\gamma}$; HER2, human epidermal growth factor receptor 2; Kit, stem cell factor receptor; EGF, epidermal growth factor.

\section{References}

1 Lyons JF, Wilhelm S, Hibner B, Bollag G. Discovery of a novel Raf kinase inhibitor. Endocr Relat Cancer 2001; 8: 219-25.

2 Awada A, Hendlisz A, Gil T, Bartholomeus S, Mano M, de Valeriola D, et al. Phase I safety and pharmacokinetics of BAY 43-9006 administered for 21 days on 7 days off in patients with advanced, refractory solid tumours. Br J Cancer 2005; 92: 1855-61.

3 Sridhar SS, Hedley D, Siu LL. Raf kinase as a target for anticancer therapeutics. Mol Cancer Ther 2005; 4: 677-85.

4 Strumberg D, Richly H, Hilger RA, Schleucher N, Korfee S, Tewes M, et al. Phase I clinical and pharmacokinetic study of the novel Raf kinase and vascular endothelial growth factor receptor inhibitor BAY 43-9006 in patients with advanced refractory solid tumors. J Clin Oncol 2005; 23: 965-72.

5 Liu L, Cao Y, Chen C, Zhang X, McNabola A, Wilkie D, et al. Sorafenib blocks the RAF/MEK/ERK pathway, inhibits tumor angiogenesis, and induces tumor cell apoptosis in hepatocellular carcinoma model PLC/ PRF/5. Cancer Res 2006; 66: 11851-8.

6 Jane EP, Premkumar DR, Pollack IF. Coadministration of sorafenib with rottlerin potently inhibits cell proliferation and migration in human malignant glioma cells. J Pharmacol Exp Ther 2006; 319: 1070-80.

7 Zhu AX. Beyond sorafenib: novel targeted therapies for advanced hepatocellular carcinoma. Expert Opin Investig Drugs 2010; 19: 663-72.

8 Wilhelm SM, Carter C, Tang L, Wilkie D, McNabola A, Rong H, et al. BAY 43-9006 exhibits broad spectrum oral antitumor activity and targets the RAF/MEK/ERK pathway and receptor tyrosine kinases involved in tumor progression and angiogenesis. Cancer Res 2004; 64: 7099-109.

9 Carlomagno F, Anaganti S, Guida T, Salvatore G, Troncone G, Wilhelm $\mathrm{SM}$, et al. BAY 43-9006 inhibition of oncogenic RET mutants. J Natl Cancer Inst 2006; 98: 326-34.

10 Aragon-Ching JB, Dahut WL. VEGF inhibitors and prostate cancer therapy. Curr Mol Pharmacol 2009; 2: 161-8.
11 Cuccuru G, Lanzi C, Cassinelli G, Pratesi G, Tortoreto M, Petrangolini G, et al. Cellular effects and antitumor activity of RET inhibitor RPI-1 on MEN2A-associated medullary thyroid carcinoma. J Natl Cancer Inst 2004; 96: 1006-14.

12 Antonelli A, Fallahi P, Ferrari SM, Mancusi C, Colaci M, Santarpia L, et al. RET TKI: potential role inthyroid cancers. Curr Oncol Rep 2012; 14: $97-104$.

13 Jhiang SM. The RET proto-oncogene in human cancers. Oncogene 2000; 19: 5590-7.

14 Takahashi M. The GDNF/RET signaling pathway and human diseases. Cytokine Growth Factor Rev 2001; 12: 361-73.

15 Wellbrock C, Hurlstone A. BRAF as therapeutic target in melanoma. Biochem Pharmacol 2010; 80: 561-7.

16 Wellbrock C, Karasarides M, Marais R. The RAF proteins take centre stage. Nat Rev Mol Cell Biol 2004; 5: 875-85.

17 Kolch W. Coordinating ERK/MAPK signalling through scaffolds and inhibitors. Nat Rev Mol Cell Biol 2005; 6: 827-37.

18 Ranieri G, Gadaleta-Caldarola G, Goffredo V, Patruno R, Mangia A, Rizzo A, et al. Sorafenib (BAY 43-9006) in hepatocellular carcinoma patients: from discovery to clinical development. Curr Med Chem 2012; 19: 938-44.

19 Molina MA, Codony-Servat J, Albanell J, Rojo F, Arribas J, Baselga J. Trastuzumab (herceptin), a humanized anti-Her2 receptor monoclonal antibody, inhibits basal and activated Her2 ectodomain cleavage in breast cancer cells. Cancer Res 2001; 61: 4744-9.

20 Heinrich MC, Corless CL, Demetri GD, Blanke CD, von Mehren M, Joensuu $\mathrm{H}$, et al. Kinase mutations and imatinib response in patients with metastatic gastrointestinal stromal tumor. J Clin Oncol 2003; 21 : 4342-9.

21 Heinrich MC, Maki RG, Corless CL, Antonescu CR, Harlow A, Griffith $D$, et al. Primary and secondary kinase genotypes correlate with the biological and clinical activity of sunitinib in imatinib-resistant gastrointestinal stromal tumor. J Clin Oncol 2008; 26: 5352-9.

22 Feld R, Sridhar SS, Shepherd FA, Mackay JA, Evans WK. Use of the epidermal growth factor receptor inhibitors gefitinib and erlotinib in the treatment of non-small cell lung cancer: a systematic review. J Thorac Oncol 2006; 1: 367-76.

23 Plaza-Menacho I, Mologni L, Sala E, Gambacorti-Passerini C, Magee Al, Links TP, et al. Sorafenib functions to potently suppress RET tyrosine kinase activity by direct enzymatic inhibition and promoting RET lysosomal degradation independent of proteasomal targeting. J Biol Chem 2007; 282: 29230-40. 